\title{
Evaluating a digital sepsis alert in a London multisite hospital network: a natural experiment using electronic health record data
}

\author{
Kate Honeyford, ${ }^{1}$ Graham S. Cooke, ${ }^{2}$ Anne Kinderlerer, ${ }^{3}$ Elizabeth Williamson, ${ }^{4}$ Mark \\ Gilchrist, ${ }^{5}$ Alison Holmes, ${ }^{6}$ The Sepsis Big Room, Ben Glampson, ${ }^{7}$ Abdulbrahim \\ Mulla, ${ }^{7}$ and Ceire Costelloe ${ }^{1}$
}

\begin{abstract}
${ }^{1}$ Global Digital Health Unit, Department of Primary Care and Public Health, Imperial College London, London, United Kingdom, ${ }^{2}$ Infectious Diseases Section, Imperial College London, London, United Kingdom, ${ }^{3}$ St Mary's Hospital, Imperial College Healthcare NHS Trust, London, United Kingdom, ${ }^{4}$ Electronic Health Records Research Group, London School of Hygiene and Tropical Medicine, London, United Kingdom, ${ }^{5}$ Department of Infectious Diseases, Imperial College Healthcare NHS Trust, London, United Kingdom, ${ }^{6}$ Health Protection Research Unit, Imperial College London, London, United Kingdom, and ${ }^{7}$ Department of Research Informatics, Imperial College Healthcare NHS Trust, London, United Kingdom

Corresponding Author: Kate Honeyford, MSc, PhD, Global Digital Health Unit, Department of Primary Care and Public Health Imperial College, Charing Cross Campus, Reynolds Building, St Dunstan's Road, London W6 8RP, UK; k.honeyford@imperial.ac.uk
\end{abstract}

Received 14 May 2019; Revised 19 July 2019; Editorial Decision 20 September 2019; Accepted 30 September 2019

\section{ABSTRACT}

Objective: The study sought to determine the impact of a digital sepsis alert on patient outcomes in a UK multisite hospital network.

Materials and Methods: A natural experiment utilizing the phased introduction (without randomization) of a digital sepsis alert into a multisite hospital network. Sepsis alerts were either visible to clinicians (patients in the intervention group) or running silently and not visible (the control group). Inverse probability of treatmentweighted multivariable logistic regression was used to estimate the effect of the intervention on individual patient outcomes.

Outcomes: In-hospital 30-day mortality (all inpatients), prolonged hospital stay ( $\geq 7$ days) and timely antibiotics ( $\leq 60$ minutes of the alert) for patients who alerted in the emergency department.

Results: The introduction of the alert was associated with lower odds of death (odds ratio, $0.76 ; 95 \%$ confidence interval [Cl], 0.70-0.84; $\mathrm{n}=21 \mathrm{183}$ ), lower odds of prolonged hospital stay $\geq 7$ days (OR, $0.93 ; 95 \% \mathrm{Cl}, 0.88-0.99$; $\mathrm{n}=9988$ ), and in patients who required antibiotics, an increased odds of receiving timely antibiotics (OR, 1.71; $95 \% \mathrm{Cl}, 1.57-1.87 ; \mathrm{n}=4622$ ).

Discussion: Current evidence that digital sepsis alerts are effective is mixed. In this large UK study, a digital sepsis alert has been shown to be associated with improved outcomes, including timely antibiotics. It is not known whether the presence of alerting is responsible for improved outcomes or whether the alert acted as a useful driver for quality improvement initiatives.

Conclusions: These findings strongly suggest that the introduction of a network-wide digital sepsis alert is asso-

(C) The Author(s) 2019. Published by Oxford University Press on behalf of the American Medical Informatics Association.

This is an Open Access article distributed under the terms of the Creative Commons Attribution Non-Commercial License (http://creativecommons.org/licenses/by-nc/4.0/), which permits non-commercial re-use, distribution, and reproduction in any medium, provided the original work is properly cited. For commercial re-use, please contact 
ciated with improvements in patient outcomes, demonstrating that digital based interventions can be successfully introduced and readily evaluated.

Key words: digital health, electronic health record, sepsis, critical care, alerts, early warning scores

\section{INTRODUCTION}

Sepsis is recognized as a common cause of serious illness and death. It is estimated that there are 123000 cases and 46000 deaths in England each year. ${ }^{1}$ Similar high levels of sepsis have been reported internationally ${ }^{2,3}$ and sepsis is recognized by World Health Organization as a global health priority. ${ }^{4}$ Many countries have nationwide sepsis action plans, and in England there are targets for hospitals to rapidly diagnose and treat patients with sepsis.

Timely, appropriately targeted, intravenous (IV) antibiotics have been shown to be effective in improving outcomes for patients, with a $4 \%$ increase in odds of mortality for every hour's delay in administration of IV antibiotics. ${ }^{5-7}$ This evidence has resulted in UK hospitals having a target (with financial incentives) of sepsis patients receiving IV antibiotics in 1 hour. ${ }^{8,9}$

To ensure rapid diagnosis and early treatment, sepsis screening tools have been introduced and refined, and include qSOFA (quick sequential organ failure assessment score), ${ }^{10}$ NEWS (National Early Warning Score), ${ }^{11}$ and NEWS2. ${ }^{12}$ Early warning scores have been shown to be effective in predicting mortality ${ }^{13}$ and intensive care unit (ICU) admission. ${ }^{14}$ There is limited evidence that the introduction of track and trigger style warning systems have been associated with improved outcomes for patients.

The introduction of electronic health records (EHRs) has provided the opportunity to embed digital alerts based on current and past clinical measurements. A range of screening algorithms have been used, including the St John Sepsis Algorithm, ${ }^{15,16}$ the Severe-Sepsis Best Practice Alert, ${ }^{17}$ and hospital-designed alerts. ${ }^{18}$ The evidence for the effectiveness of these alerts on patient outcomes is mixed. ${ }^{19}$ Some studies have shown that introduction of digital sepsis alerts have led to increases in the proportion of patients with suspected sepsis receiving IV antibiotics in 1 hour, ${ }^{17}$ reduced ICU and hospital length of stay (LOS), ${ }^{20}$ and reduced in-hospital mortality, ${ }^{18,20}$ while others have shown no significant effect on $\operatorname{LOS}^{20,21}$ or in-hospital mortality. ${ }^{17} \mathrm{~A}$ recent randomized controlled trial (RCT) analyzing the impact of the introduction of an alert for inpatients in a U.S. hospital found no association between the introduction of an alert and an improvement in patient outcomes, although the study was terminated when the hospital decided to roll the alert out, and hence underpowered to detect associations. $^{22}$ The majority of evidence comes from studies in the U.S. healthcare system and sample sizes have been relatively small. It is not known if similar impacts on patient outcomes will be seen in larger scale studies, particularly in an English hospital, where care is free at the point of delivery and accessible to all.

The aims of this study were to determine the effect of the introduction of a digital sepsis alert on 1) key process measure (timely antibiotics); and 2) patient outcomes (extended LOS and in-patient 30-day mortality)

\section{MATERIALS AND METHODS}

\section{Study design}

In this natural experiment, a weighted multiple logistic regression was used to examine the effect of the digital sepsis alert. Data from
October 2016 to May 2018 was included in the study which utilized a "silent" running phase, during which time digital alerts were active but not visible to clinicians. The silent phase provides a control group. Robust statistical methods were used to balance characteristics between the live and control phases. The primary outcome was 30-day inpatient mortality and a secondary outcome of prolonged LOS ( $\geq 7$ days). Additionally, the impact of the introduction of the alert on the key process measure of timely IV antibiotics ( $\leq 60$ minutes after the alert) was studied.

\section{Digital sepsis alert}

The digital sepsis alert is based on the St John Sepsis Algorithm developed by Cerner Corporation, ${ }^{26}$ shown in Figure 1 . The alert is an integrated part of the EHR and has a silent running mode. Silent alerts are not visible to clinical staff at the "front end" of the system. Once the alert is turned on (live) in a clinical area nurses and doctors are notified of patients who have triggered the alert. Nurses are notified of patients who have triggered the alert either through a pop-up warning on the EHR (in inpatient wards) or as a dashboard which highlights any patient with an active alert (in the emergency department [ED] and inpatient wards). Doctors are presented with a sepsis warning only when they open the patient's record. In addition to the alert, a novel multidisciplinary care pathway, designed by the Trust, launches from the digital record when the clinician confirms suspicion of sepsis. These "Treatment Plans" support the clinician to start treatment in-line with hospital guidance, including fluids, oxygen, diagnostic tests (blood and other cultures), and early antibiotics. Content from local infection guidelines is built in, so that for any given potential sepsis diagnosis, the appropriate antibiotics with appropriate dosing and directions are prompted.

The introduction of the alerts was part of a framework for system redesign and improvement, see Box 1 for more details. The alert was introduced in a phased approach over an 18-month period across the Trust, summarized in Figure 2. The alert was switched from silent to live as recommended by improvement approaches for scale and spread. ${ }^{25,27}$ Initially in the acute medical unit at one site, expanding out to both EDs and hematology wards and then Trust-wide. Initial areas were selected based on their interest in assistance in identifying patients with suspicion of sepsis.

\section{Patient population}

Patients who triggered the alert were included in the analysis. These are patients who were identified as potentially having sepsis by the clinical thresholds included in the St John's Sepsis alert (Figure 1). The unit of analysis was an adult inpatient "encounter." An encounter was defined as a continuous spell in the Trust. In this analysis all encounters of adult patients ( 18 years of age and older) in which a sepsis alert was triggered were included. Although the alert may be triggered repeatedly for a patient during a hospital encounter, only first alerts were considered. All patients admitted to the 3 hospitals in the network that have general acute admissions were included. 


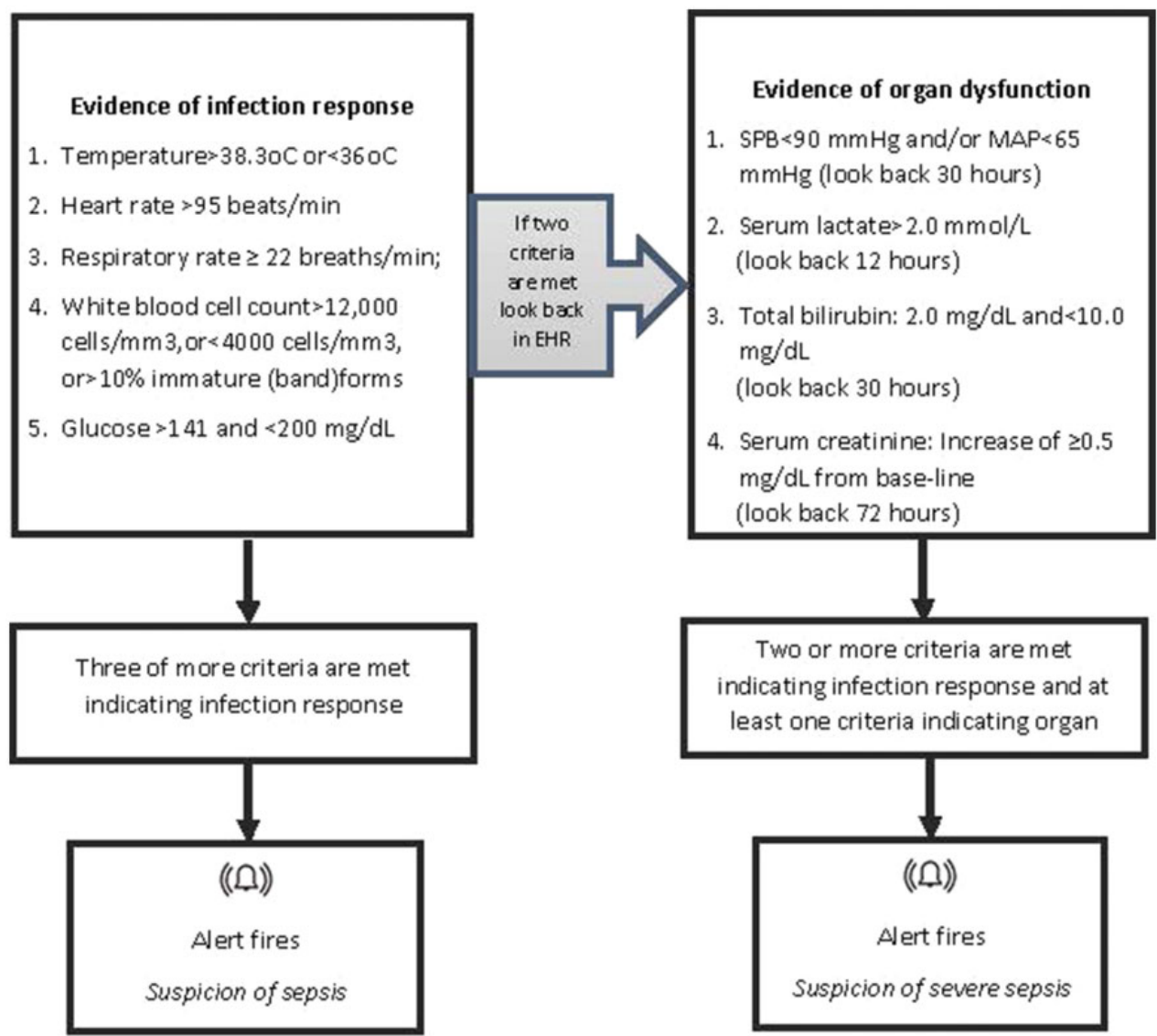

Figure 1. Criteria associated with the St John Sepsis Alert. ${ }^{23}$ MAP: Mean Arterial Pressure; SPB: Systolic Blood Pressure.

Only encounters ending in admission were included in final models to ensure detailed comorbidity and outcome information were available. For LOS and timely antibiotics, only patients who alerted in the ED were included, to reduce potential confounders including the high proportion of inpatients who are on antibiotics when the alert fires for reasons not directly related to the alert and the impact of prior long inpatient stays on LOS postinfection. Patients who were already on antibiotics were excluded from the analysis of timely antibiotics. Patients who did not receive antbiotics within 24 hours of the alert were excluded-it was assumed the alert had triggered for patients who did not require antibiotics (further details in Supplementary Appendix S1). See Figure 3 for a summary of patient cohorts.

\section{Outcomes}

The outcomes were (1) in-hospital all-cause mortality within 30 days of alert, (2) long hospital stay ( $\geq 7$ days), and (3) timely antibiotics (IV antibiotics $\leq 60$ minutes). Both long hospital stay and timely antibiotics were investigated only for patients who alerted in the ED. LOS was measured as time from alert to discharge.

For the purposes of this study, timely antibiotics was defined as patients who received IV antibiotics within 1 hour of the alert. This definition was informed by the current target for hospitals in England. ${ }^{8}$

\section{Statistical analysis}

Three separate analyses were undertaken in 3 cohorts to explore the 3 main outcomes. The switch from silent to live was considered as a natural experiment. Inverse probability of treatment weighting (IPTW) was used ${ }^{29}$ to account for confounding the nonrandom allocation introduced and balance characteristics between the live and control phases. Multivariable logistic regression was used to determine the propensity score weights. As the sample was different for each outcome, separate regression models were used to determine propensity score weight observations in each sample. Further details are available in Supplementary Appendix S2. Potential confounders for the three outcomes and alert status allocation were included in models. These included patient characteristics, admission details and clinical measures. Data were obtained from patient digital medical records. Details of variables are included in Box 2.

Balance between treatment populations was evaluated using standardized mean differences (SMDs) of all baseline covariates. A threshold of $10 \%$ indicates possible imbalance and $25 \%$ was an indication of unacceptable imbalance.

The odds ratio (OR) and $95 \%$ confidence interval $(\mathrm{CI})$ were estimated for each outcome using logistic regression applying the propensity score weights. A doubly robust approach was employed, ${ }^{30}$ including covariates in both the propensity score models and the multivariable logistic models of the outcomes. When modeling death, a random-effects model was used to account for clustering within the acute, hematology, and ED areas, with all other alerts included in an additional cluster. Additionally the outcomes were modeled using logistic regression without applying the propensity score weights but adjusting for confounders. All analyses were done with $\mathrm{R}$ version 3.2.3 (R Foundation for Statistical Computing, Vienna, Austria). 


\section{BOX 1. The local picture.}

The hospital network (Trust) comprises of 5 main sites. In recent years, the Trust had over 1 million outpatient contacts, quarter of a million ED attendances, 200000 inpatient contacts, and 100000 inpatient operations. The Trust employs more than 2500 doctors, 4000 nurses, 720 allied healthcare professionals, and 130 pharmacists.

Work to improve care for sepsis patients at the Trust centers on 3 key priorities:

- The identification and treatment of sepsis across the whole patient pathway

- Consistency of standards and reporting

- The prudent use of antimicrobials within the wider antimicrobial stewardship and resistance agenda.

A key focus has been to ensure that patients identified with sepsis receive the appropriate antibiotics within 1 hour, in line with national targets. The work is integrated with the digital transformation and the use of an embedded digital sepsis alert in the EHR.

The digital sepsis alert embedded in the EHR and available to the Trust is the St John Sepsis Algorithm.

Implementation of the alert was part of a collaborative improvement approach through the Sepsis Big Room. A "big room" is a weekly coached meeting which provides time and space for a range of staff to come together to discuss improvements to the quality of patient care. Staff from all disciplines are welcome and the meetings operate a flattened hierarchy. Patient stories are reviewed and real-time data displayed to support the identification of specific improvements to healthcare processes within the pathway of care. In an approach similar to one others have used, a series of tests of change were undertaken to improve decision making and communication for sepsis patients. For each test, a small-scale Plan-DoStudy-Act cycle, based on Toyota Big Room methodology, ${ }^{23,24}$ was performed and, if this proved successful, the test was tried more widely. Data from the evaluation were used to provide feedback to the team and shape discussion on implementation. A key aspect of work undertaken by the Sepsis Big Room was the development of "Treatment Plans" for specific diagnosis which are embedded in the EHR system. A sepsis treatment plan was developed, which includes relevant investigations, fluids, oxygen prescriptions, and specific antibiotics according to guidelines. This is in line with the Institute for Healthcare Investigations sepsis bundle. ${ }^{25}$

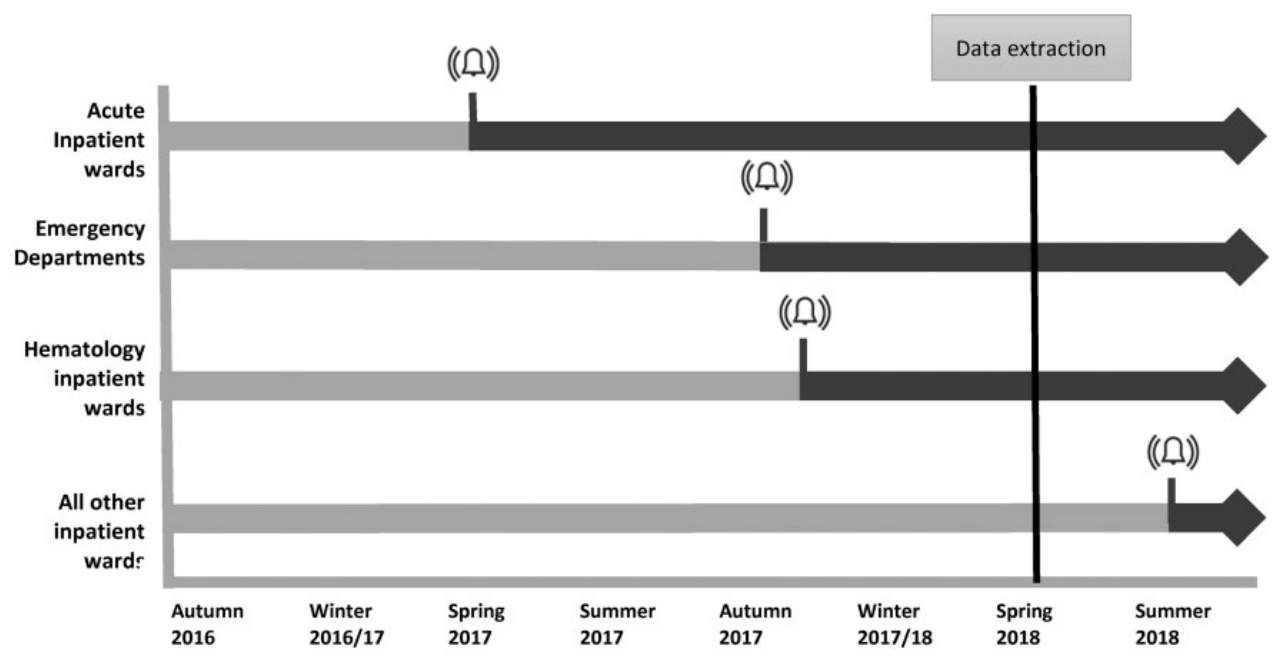

Figure 2. Phased introduction of live alerts across multisite hospital from autumn 2016. The digital alert was switched from silent to live in acute wards, followed by emergency departments in 2 hospital sites in autumn and hematology departments soon after. The alert was switched to live across all inpatient wards in August 2018, after data were extracted for this study.

\section{RESULTS}

\section{Study population}

In total, there were 21732 patient encounters with at least 1 alert between October 2016 and May 2018. A total of 9988 of these were in the ED, 942 alerted in acute wards, and 1218 alerted in hematology wards. A total of $4622 \mathrm{ED}$ patients were not on IV antibiotics at the time of the alert and did receive IV antibiotics within 24 hours of the alert. See Figure 3 for cohort details.
Table 1 summarizes the characteristics of patients for whom the alert was during a silent phase and those for whom the alert was in a live phase (clearly visible to clinicians). The phased introduction of the live alert means that the patient and encounter characteristics of the 2 groups are not balanced and there are more live alerts for patients admitted to site $\mathrm{C}$, admitted through the ED, and admitted in the autumn and winter. Within live alerts, there was a higher proportion of younger patients (18-44 years of age) and older patients (85 years of age and older). In comparison with silent 


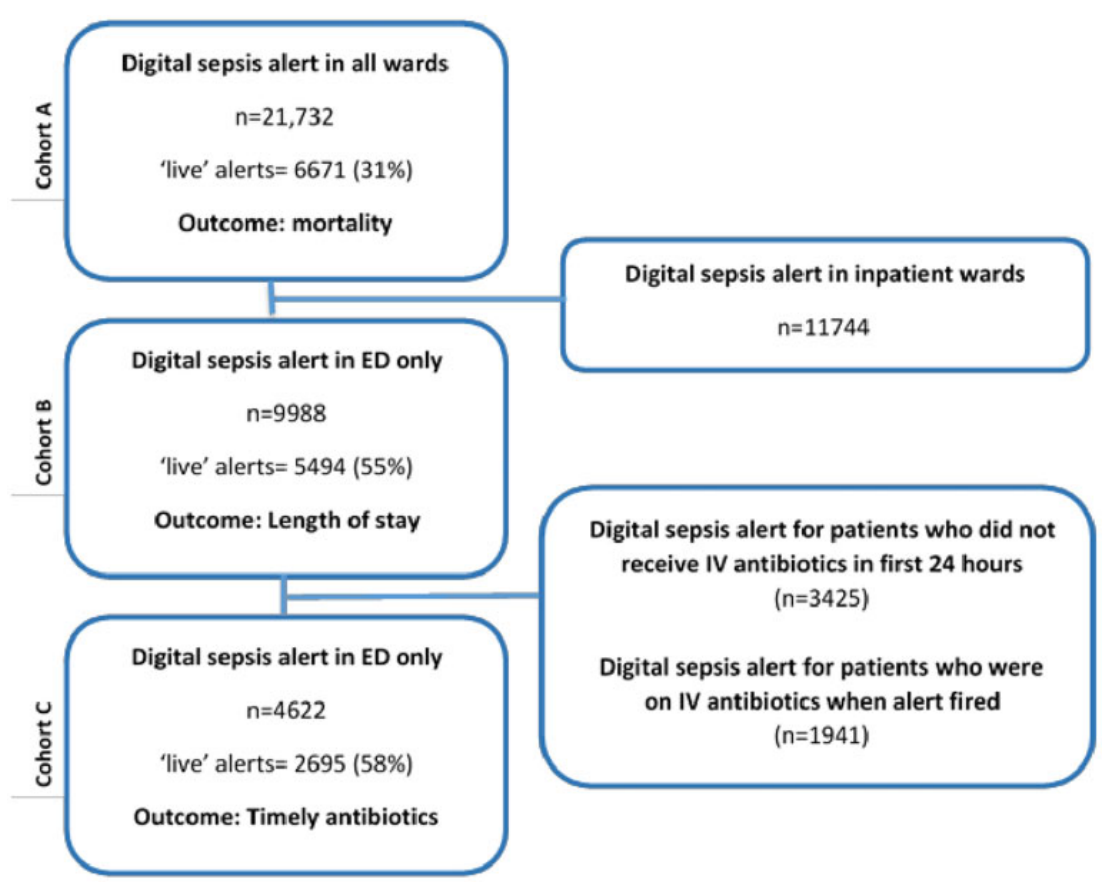

Figure 3. Cohort definition. Three cohorts were developed to investigate the outcomes of interest. Cohort A comprised all patients who alerted and the outcome of interest in this cohort was mortality. Cohort B comprised patients who alerted in the emergency departments (EDs) only, as the main outcome of interest was length of stay. Cohort $\mathrm{C}$ comprised patients who alerted in the ED who received antibiotics within 24 hours postalert. The main outcome of interest was timely antibiotics, defined a receiving antibiotic within 1 hour of the alert (as per NICE guidelines). ${ }^{28}$

running, patients who alerted in the live phase were more likely to have pulmonary conditions but less likely to have renal conditions. In addition, these patients were less likely have an unknown ethnicity. A higher proportion of patients who alerted in the live phase had medium, high, or missing NEWS score. A higher proportion had suspected severe sepsis in comparison to suspected sepsis.

IPTW was used to account for these baseline differences. Models were adjusted for differences in the 2 groups using IPTW. After weighting, all models were balanced. SMDs for cohort $\mathrm{B}$ used to model LOS and cohort $\mathrm{C}$ used to model timely antibiotics were $3.0 \%$ or less (compared with a highest value of over $70 \%$ before imposing estimated weights). For cohort A, to model death, the SMDs for hospital site, alert introduction area (cluster), age, NEWS, diabetes, and renal disease were all above $10 \%$ but under $25 \%$. As a doubly robust method all potential confounders were included in the final model accounting for any confounding from known confounders. Further details are available in Supplementary Appendix S3.

\section{Association of alert status with death}

A total of 21732 inpatients alerted during the period of study, across all wards, across the 3 sites. A total of 1293 (6.0\%) patients died within 30 days of the alert being triggered, which is similar to mortality rates reported elsewhere. ${ }^{21}$ Live alert status was associated with lower in-hospital mortality $(5.1 \%$ compared with $6.4 \%$; $P<.001$ ) (see Table 2). After accounting for patient characteristics, using IPTW propensity scores and patient characteristics in the multivariable logistic model, patients who alerted during the live phase had $24 \%$ lower odds (95\% CI, 16\%-30\% lower odds) of in-hospital death.

\section{Association of alert status with long LOS}

A total of 9988 patients alerted in the ED and were subsequently admitted and $4055(40.6 \%)$ of these patients subsequently had a long $\operatorname{LOS}(\geq 7$ days), measured as time from the alert to discharge. After accounting for patient characteristics, live alert status was significantly associated with decreased odds of long LOS. Patients who alerted during the live phase had a $7 \%$ lower odds of a long LOS.

\section{Association of alert status with timely antibiotics}

A total of 6563 of the $9988(65.7 \%)$ patients who alerted in the ED received antibiotics within \pm 24 hours of the alert firing.

Of the 4622 patients who were not on antibiotics when the alert fired, $36.9 \%$ of encounters that activated during the control period resulted in IV antibiotics administered within 1 hour of the alert and $44.7 \%$ of encounters activated the alert when it was visible to clinicians. After accounting for patient characteristics, live alert status was associated with $71 \%$ higher odds of receiving timely antibiotics (OR, 1.71; 95\% CI, 1.57-1.87). This approximates to a relative risk of 1.35 (95\% CI, 1.28-1.41), a 35\% increase in chance of receiving timely IV antibiotics.

Patients who did not receive antibiotics are summarized in the Supplementary Appendix Table S1A. It is assumed that the majority of these patients did not need IV antibiotics. This assumption is supported by the improved prognosis of these patients $(6.4 \%$ died and $46.6 \%$ had a prolonged LOS compared with $3.8 \%$ and $29.1 \%$ of those who did not receive antibiotics). Patient characteristics were compared in those that received antibiotics and those that did not. A higher proportion of elderly patients, patients with a high NEWS score, and patients who alerted in the spring and winter received antibiotics. Ethnicity, deprivation, and sex were not significantly associated with receipt of antibiotics, suggesting that 
BOX 2. Covariates included in both the propensity score models and the multivariable logistic models.

\begin{tabular}{|c|c|}
\hline & Patient characteristics \\
\hline Age & Grouped into $18-44 ; 45-64 ; 65-69 ; 70-74 ; 75-79 ; 80-84 ;$ and $85+$ years of age \\
\hline Sex & Male or female \\
\hline \multirow[t]{2}{*}{ Ethnicity } & Based on the following groupings: \\
\hline & White; Asian; Black; other; and not known. \\
\hline \multirow[t]{2}{*}{ Comorbidities } & $\begin{array}{l}\text { Based on any relevant International Classification of Diseases-Tenth Revision code appearing in the dis- } \\
\text { charge diagnosis codes ( } 25 \text { possible codes) }\end{array}$ \\
\hline & $\begin{array}{l}\text { Myocardial infarction; congestive heart failure; peripheral vascular disease; stroke; dementia; pulmonary; } \\
\text { rheumatic; peptic ulcer disease; liver (mild); liver (severe); diabetes; diabetes (complex); paralysis; re-- } \\
\text { nal; metastatic cancer; human immunodeficiency virus. }\end{array}$ \\
\hline \multirow[t]{2}{*}{$\begin{array}{l}\text { Deprivation } \\
\text { quintile }\end{array}$} & $\begin{array}{l}\text { Measured as the deprivation score of the patient's primary care practice, obtained by matching patients } \\
\text { to their registered practice. If patients did not have a registered primary care practice or the practice } \\
\text { was not included in the Public Health England practice profiles a "missing" categorization was allo- } \\
\text { cated. There are therefore } 6 \text { deprivation categories, with Quintile } 1 \text { being the least deprived. }\end{array}$ \\
\hline & Admission characteristics \\
\hline \multirow{3}{*}{$\begin{array}{r}\text { Admitting } \\
\text { hospital }\end{array}$} & A has an ED department \\
\hline & B no ED department \\
\hline & C has an ED department \\
\hline \multirow{5}{*}{$\begin{array}{l}\text { Season of } \\
\text { admission }\end{array}$} & Spring: March, April, May \\
\hline & Summer: June, July, August \\
\hline & Autumn: September, October, November \\
\hline & Winter: December, January, February \\
\hline & Patient severity \\
\hline \multirow[t]{6}{*}{ NEWS score } & Categorized into: zero, low, medium, high, and none recorded \\
\hline & NEWS $=0:$ zero \\
\hline & $1 \leq \mathrm{NEWS}<5:$ low \\
\hline & $5 \leq \mathrm{NEWS}<7:$ medium \\
\hline & $7 \geq$ NEWS: high \\
\hline & A NEWS score is available for $19599(90 \%)$ of the patients. \\
\hline \multirow[t]{2}{*}{ Alert } & Suspected sepsis \\
\hline & Suspected severe sepsis \\
\hline
\end{tabular}

clinical aspects of the patient and not the underlying health inequalities are associated with receipt of antibiotics. Sensitivity analysis was carried out to determine the impact of excluding patients who had not received antibiotics within 24 hours, and when these were included the introduction of the live alert was associated with a $81 \%$ higher odds of receiving timely antibiotics (OR, 1.81; 95\% CI, 1.68-1.96).

\section{DISCUSSION}

This is the first evaluation of a digital sepsis alert in an English hospital and the largest undertaken anywhere to date. Robust methods were used to show that the introduction of a digital sepsis alert was associated with improvements in patient outcomes. Overall, $6.0 \%$ of patients who alerted as possible sepsis patients died within 30 days of the alert (all-cause, in-hospital); this is in a hospital network with a lower than expected overall in-hospital mortality. ${ }^{31}$ Patients for whom their first alert was during the live phase had lower odds of death (OR, 0.76; 95\% CI, 0.70-0.84), lower odds of a long hospital stay ( $\geq 7$ days) (OR, 0.93; 95\% CI, 0.88-0.99), and increased odds of receiving timely antibiotics (OR, 1.71; 95\% CI, 1.57-1.87). The magnitude and interpretation of these results is similar when using either a weighted or unweighted multiple logistic regression model. These results suggest an important clinical benefit from the introduction of alerting, although it is not possible to say the extent to which the presence of alerting, per se, is responsible for the benefits seen, or whether the alert acted as a useful driver for other quality improvement initiatives.

The phased nature of the introduction of the alert allows a detailed analysis, with live alerts and controls coming from a range of wards, settings, and time periods. Previous studies have not given a consistent picture of the effectiveness of electronic sepsis alerts on improving patient outcomes. Reasons for this may include the variation in patient cohorts studied, that is, ICU patients, ${ }^{18} \mathrm{ED}$ patients, ${ }^{32}$ patients with a confirmed sepsis diagnosis, or all alerting patients as in this study. Studies also vary in their choice of outcomes, delivery of the complete 3-hour sepsis bundle, which includes therapeutic and diagnostic steps, is commonly reported in U.S. studies; see Shah et $\mathrm{al}^{33}$ as an example. LOS is also reported, but modeled in a variety of ways, from 72 hours $^{22}$ to analyzing mean differences. ${ }^{21}$ Many studies are small with $<500$ patients, ${ }^{17,22,33}$ which means that statistical power to detect less common outcomes, such as mortality, is low. Furthermore, the majority of studies are observational studies and robust approaches to accounting for selection bias and time trends are not commonly used, although these were addressed by Austrian et al. ${ }^{32}$ 
Table 1. Distribution of patient and encounter characteristics for all alerts and standardized mean difference before and after weighting

\begin{tabular}{|c|c|c|c|c|c|c|c|c|c|}
\hline \multirow[t]{2}{*}{ Factor } & \multirow[t]{2}{*}{ Level } & \multicolumn{2}{|c|}{$\begin{array}{c}\text { Control phase } \\
\text { alerts } \\
(\mathrm{n}=15056)\end{array}$} & \multicolumn{2}{|c|}{$\begin{array}{l}\text { Live phase } \\
\quad \text { alerts } \\
(\mathrm{n}=6127)\end{array}$} & \multicolumn{2}{|c|}{$\begin{array}{c}\text { All alerts } \\
(\mathrm{n}=21 \text { 183) }\end{array}$} & \multicolumn{2}{|c|}{$\begin{array}{l}\text { Standardized } \\
\text { mean } \\
\text { difference }(\%)\end{array}$} \\
\hline & & $\mathrm{n}$ & $\%$ & $\mathrm{n}$ & $\%$ & $\mathrm{n}$ & $\%$ & Before IPTW & After IPTW \\
\hline \multirow[t]{7}{*}{ Age group } & $18-44$ y (ref) & 2588 & 17.2 & 1239 & 20.2 & 3827 & 18.1 & 13.0 & 11.5 \\
\hline & $45-64 y$ & 4431 & 29.4 & 1644 & 26.8 & 6075 & 28.7 & & \\
\hline & $65-69 y$ & 1594 & 10.6 & 515 & 8.4 & 2109 & 10.0 & & \\
\hline & $70-74 y$ & 1583 & 10.5 & 603 & 9.8 & 2186 & 10.3 & & \\
\hline & $75-79 y$ & 1605 & 10.7 & 636 & 10.4 & 2241 & 10.6 & & \\
\hline & $80-84$ y & 1393 & 9.3 & 601 & 9.8 & 1994 & 9.4 & & \\
\hline & $85 \mathrm{y}$ and older & 1862 & 12.4 & 889 & 14.5 & 2751 & 13.0 & & \\
\hline \multirow[t]{2}{*}{ Sex } & Female & 6936 & 46.1 & 2735 & 44.6 & 9671 & 45.7 & 2.0 & 0.4 \\
\hline & Male & 8120 & 54.0 & 3392 & 55.4 & 11512 & 54.4 & & \\
\hline \multirow[t]{5}{*}{ Ethnicity } & White & 6986 & 46.4 & 3063 & 50.0 & 10049 & 47.4 & 12.8 & 2.7 \\
\hline & Black & 1667 & 11.1 & 708 & 11.6 & 2375 & 11.2 & & \\
\hline & Not known & 2473 & 16.4 & 784 & 12.8 & 3257 & 15.4 & & \\
\hline & Other & 2923 & 19.4 & 1257 & 20.5 & 4180 & 19.7 & & \\
\hline & Asian & 1007 & 6.7 & 315 & 5.1 & 1322 & 6.2 & & \\
\hline \multirow[t]{6}{*}{ Deprivation quintile } & Least deprived & 2787 & 18.5 & 1591 & 26.0 & 4378 & 20.7 & 22.4 & 9.1 \\
\hline & & 3953 & 26.3 & 1662 & 27.1 & 5615 & 26.5 & & \\
\hline & & 4024 & 26.7 & 1468 & 24.0 & 5492 & 25.9 & & \\
\hline & & 2243 & 14.9 & 713 & 11.6 & 2956 & 14.0 & & \\
\hline & Most deprived & 1277 & 8.5 & 319 & 5.2 & 1596 & 7.5 & & \\
\hline & Not known & 772 & 5.1 & 374 & 6.1 & 1146 & 5.4 & & \\
\hline \multicolumn{2}{|l|}{ Myocardial infarction } & 1755 & 11.7 & 579 & 9.5 & 2334 & 11.0 & 7.2 & 8.4 \\
\hline \multicolumn{2}{|l|}{ Heart failure } & 2420 & 16.1 & 858 & 14.0 & 3278 & 15.5 & 5.9 & 9.4 \\
\hline \multicolumn{2}{|c|}{ Peripheral vascular disease } & 1186 & 7.9 & 384 & 6.3 & 1570 & 7.4 & 6.9 & 1.2 \\
\hline \multicolumn{2}{|l|}{ Stroke } & 2257 & 15.0 & 909 & 14.8 & 3166 & 15.0 & 0.8 & 1.7 \\
\hline \multicolumn{2}{|l|}{ Dementia } & 1082 & 7.2 & 558 & 9.1 & 1640 & 7.7 & 8.0 & 1.8 \\
\hline \multicolumn{2}{|l|}{ Pulmonary } & 3626 & 24.1 & 1928 & 31.5 & 5554 & 26.2 & 16.0 & 1.5 \\
\hline \multicolumn{2}{|l|}{ Rheumatic } & 567 & 3.8 & 225 & 3.7 & 792 & 3.7 & 0.3 & 4.0 \\
\hline \multicolumn{2}{|l|}{ Peptic ulcer disease } & 215 & 1.4 & 109 & 1.8 & 324 & 1.5 & 1.8 & 7.3 \\
\hline Liver disease-mild & & 957 & 6.4 & 426 & 7.0 & 1383 & 6.5 & 2.0 & 0.6 \\
\hline Diabetes-uncomplicat & & 3700 & 24.6 & 1478 & 24.1 & 5178 & 24.4 & 1.1 & 4.6 \\
\hline Diabetes-complicated & & 1145 & 7.6 & 340 & 5.6 & 1485 & 7.0 & 8.8 & 11.3 \\
\hline Paralysis & & 649 & 4.3 & 243 & 4.0 & 892 & 4.2 & 1.9 & 4.8 \\
\hline Renal & & 3141 & 20.9 & 801 & 13.1 & 3942 & 18.6 & 21.6 & 14.2 \\
\hline Liver disease-severe & & 328 & 2.2 & 160 & 2.6 & 488 & 2.3 & 1.9 & 0.6 \\
\hline Metastatic cancer & & 1329 & 8.8 & 348 & 5.7 & 1677 & 7.9 & 10.6 & 1.2 \\
\hline Human immunodefici & ncy virus & 150 & 1.0 & 64 & 1.0 & 214 & 1.0 & 0.5 & 3.1 \\
\hline Trust site & A & 6342 & 42.1 & 2427 & 39.6 & 8769 & 41.4 & 48.3 & 11.5 \\
\hline & $\mathrm{B}$ & 4684 & 31.1 & 872 & 14.2 & 5556 & 26.2 & & \\
\hline & $\mathrm{C}$ & 4030 & 26.8 & 2828 & 46.2 & 6858 & 32.4 & & \\
\hline Season of admission & Spring & 2359 & 15.7 & 2173 & 35.5 & 4532 & 21.4 & 58.8 & 6.0 \\
\hline & Summer & 2608 & 17.3 & 394 & 6.4 & 3002 & 14.2 & & \\
\hline & Autumn & 5553 & 36.9 & 1380 & 22.5 & 6933 & 32.7 & & \\
\hline & Winter & 4536 & 30.1 & 2180 & 35.6 & 6716 & 31.7 & & \\
\hline Severity & Suspected sepsis (ref) & 8025 & 53.3 & 2775 & 45.3 & 10800 & 51.0 & 10.3 & 3.1 \\
\hline & Suspected severe sepsis & 7031 & 46.7 & 3352 & 54.7 & 10383 & 49.0 & & \\
\hline NEWS score & Zero (ref) & 617 & 4.1 & 183 & 3.0 & 800 & 3.8 & 23.3 & 17.7 \\
\hline & Low & 8513 & 56.5 & 2887 & 47.1 & 11400 & 53.8 & & \\
\hline & Medium & 2331 & 15.5 & 1165 & 19.0 & 3496 & 16.5 & & \\
\hline & High & 2277 & 15.1 & 1208 & 19.7 & 3485 & 16.5 & & \\
\hline & Missing & 1318 & 8.8 & 684 & 11.2 & 2002 & 9.5 & & \\
\hline
\end{tabular}

IPTW: inverse probability of treatment weighting;NEWS: National Early Warning Score. 
Table 2. Summary data and results of models, including adjustment for confounders

\begin{tabular}{|c|c|c|c|c|c|c|}
\hline & \multicolumn{2}{|c|}{ Death } & \multicolumn{2}{|c|}{ Extended LOS } & \multicolumn{2}{|c|}{ Timely Antibiotics } \\
\hline & Control & Live & Control & Live & Control & Live \\
\hline Total encounters & 15061 & 6671 & 4494 & 5494 & 1927 & 2695 \\
\hline Number of events & 959 & 339 & 1846 & 2209 & 712 & 1204 \\
\hline \multirow[t]{2}{*}{$\%$ events } & 6.4 & 5.1 & 41.1 & 40.2 & 36.9 & 44.7 \\
\hline & \multicolumn{2}{|c|}{ OR $(95 \%$ CI $)$} & \multicolumn{2}{|c|}{ OR $(95 \% \mathrm{CI})$} & \multicolumn{2}{|c|}{ OR $(95 \%$ CI $)$} \\
\hline Unadjusted & \multicolumn{2}{|c|}{$0.67(0.67-0.90)$} & \multicolumn{2}{|c|}{$0.97(0.89-1.05)$} & \multicolumn{2}{|c|}{$1.38(1.22-1.55)$} \\
\hline Adjusted (reg) ${ }^{\mathrm{a}}$ & \multicolumn{2}{|c|}{$0.79(0.67-0.93)$} & \multicolumn{2}{|c|}{$0.97(0.87-1.05)$} & \multicolumn{2}{|c|}{$1.70(1.43-1.95)$} \\
\hline Adjusted (IPTW) ${ }^{\mathrm{b}}$ & \multicolumn{2}{|c|}{$0.76(0.70-0.84)$} & \multicolumn{2}{|c|}{$0.93(0.88-0.99)$} & \multicolumn{2}{|c|}{$1.71(1.57-1.87)$} \\
\hline & \multicolumn{2}{|c|}{$\operatorname{RR}(95 \% \mathrm{CI})^{\mathrm{c}}$} & \multicolumn{2}{|c|}{$\operatorname{RR}(95 \% \mathrm{CI})^{\mathrm{c}}$} & \multicolumn{2}{|c|}{$\operatorname{RR}(95 \% \mathrm{CI})^{\mathrm{c}}$} \\
\hline Adjusted (IPTW) ${ }^{\mathrm{b}}$ & \multicolumn{2}{|c|}{$0.76(0.70-0.84)^{\mathrm{d}}$} & \multicolumn{2}{|c|}{$0.96(0.93-0.99)$} & \multicolumn{2}{|c|}{$1.35(1.28-1.41)$} \\
\hline
\end{tabular}

After adjustment for potential confounding and IPTW, measures of association did not change markedly, but were more precise.

CI: confidence interval; IPTW: inverse probability of treatment weighting; LOS: length of stay; OR: odds ratio; RR: relative risk.

${ }^{a}$ Adjusted for all confounders summarized in Table 1.

b Propensity score-weighted log-linear fully adjusted model used to estimate Odds Ratios.

${ }^{c}$ Relative risks determined using a fully adjusted, propensity score-weighted log-linear model.

${ }^{\mathrm{d}}$ With the exception of death, which is estimated directly from the OR, as the event is rare.

A recent RCT found that the introduction of a sepsis alert had no impact on receipt of antibiotics within 3 hours or additional outcomes, including in-hospital mortality. ${ }^{22}$ Possible reasons for the lack of effects suggested by the authors include a baseline high compliance and the alert firing after clinicians had diagnosed patients. In addition, this RCT focuses on a different patient cohort to our study including patients in wards, not in the ED, and further work is necessary to determine if alerts have different impacts in different settings. The RCT was terminated early when the hospital quality committee decided to roll out the alert to all eligible patients, meaning that the study did not have the planned statistical power to detect change. A study by Austrian et $\mathrm{al}^{32}$ did not find any impact on patient outcomes when an alert was introduced in an ED, although there was a significant impact on LOS. Smaller studies in the United States have found interventions which included an alert triggered by an EHR based diagnosis resulted in improved outcomes for patients. McRee et $\mathrm{al}^{21}$ found a reduction in mortality from $9.3 \%$ to $1.0 \%$ but no significant effect on LOS, although this was a small pilot study $(\mathrm{n}=171)$. In another small study $(\mathrm{n}=214)$ in the United States, the introduction of an alert was associated with an improvement in patients receiving timely antibiotics, from $48.6 \%$ to $76.7 \%$, and a significant reduction in LOS. ${ }^{17}$ Shah et $\mathrm{al}^{33}$ also found an increase in the receipt of antibiotics within one hour, although they did not take into account any trends in antibiotic administration in their pre- and postphase analysis. There was no significant impact on in-hospital mortality. Westra et $\mathrm{al}^{34}$ and Guirgis et $\mathrm{al}^{35}$ found the introduction of an alert, bundled with education, training, and structured care sets, to be associated with reductions in mortality and LOS.

This analysis of a large sample of patients who have been admitted across 3 sites to a busy hospital network in England is one of the largest to date. For mortality, both patients who presented with suspected sepsis in the ED and those who developed symptoms congruent with sepsis during their inpatient stay were included. For LOS and timely antibiotics, patients who alerted in the ED and were subsequently admitted were included in the analysis. Outcomes were selected based on their importance to both patients and hospitals, including those based on UK government targets, and were applied to all patient encounters where there was an alert, not limited to patients who were confirmed as having sepsis. A key methodological strength of this study was the inclusion of a "silent" running phase, during which time digital alerts were active but not visible to clinicians. The silent phase provides a control group. In addition, robust statistical methods were used to balance characteristics between the live and control phases.

There are a number of limitations to our study. First, the quasiexperimental design limits ability to imply causation. Although RCTs are considered the gold standard for analyzing health interventions, this approach was not deemed possible in the complex environment of this busy, multisite hospital. Difficulties of conducting an RCT on digital alerts for sepsis have been documented elsewhere. $^{22}$ Statistical approaches recommended for the analysis of data derived from natural experiment were used. ${ }^{36}$ Propensity scores are a recognized and recommended method to adjust for confounding factors, in this case introduced by the phased introduction of the alert. The majority of live alerts were ED patients who attended in autumn and winter, which resulted in a higher proportion of severe sepsis and higher NEWS scores in the live group. The impact of the wider sepsis quality improvement initiatives on improved outcomes for patients could not be robustly modeled, as it was not possible to associate the introduction of these initiatives with specific periods of time. Aspects of our analysis were limited by data availability; only admitted patients were included because clinical information was limited for patients discharged from the ED without admission. The analysis of the association of the alert on timely antibiotics and LOS was limited to patients in the ED to reduce potential confounding caused by patients being on antibiotics for reasons unconnected to the sepsis alert and the impact of prior long inpatient stays on LOS postinfection. We only found a significant impact on LOS after applying weights to correct in-balance between the 2 samples. Our definition of extended LOS was informed by national policy in England. Different definitions of LOS leading to different categorizations may have resulted in different interpretation of the impact of the alert.

In this study we have not considered unintended consequences of the introduction of the alert, including the possibility of increases in the use of inappropriate or unnecessary IV antibiotics, increases in patients being diagnosed as having sepsis without confirmation, and the possibility of alert fatigue as a result of relatively low specificity of the alert. This is the focus of future work. 
The introduction of digital altering for sepsis is an opportunity to improve care for patients who may have sepsis. Despite the current emphasis on the use of sepsis screening tools, including the recommendation of the uptake of NEWS2 as the best current option for standardizing the management of deterioration and sepsis, ${ }^{37}$ there is uncertainty around the use of digital screening to improve patient outcomes. ${ }^{38}$ This study, the largest to date, is an important addition to the body of knowledge of appropriate digital-based screening, and shows that when associated with quality improvement approaches is associated with improved patient outcomes.

\section{CONCLUSION}

In 2 busy acute hospitals, the introduction of a digital sepsis alert has been shown to be associated with improved patient outcomes, including lower risk of mortality and extended LOS. A 70\% increase in odds of receiving timely antibiotics was found, which is likely to be important in explaining the causal pathway for the alert improving outcomes for patients. This study has clearly shown that the introduction of a network-wide digital screening tool embedded in EHRs is associated with improvement in patient outcomes, demonstrating that digital-based interventions can be successfully introduced and readily evaluated.

\section{FUNDING}

This report is independent research funded by the National Institute for Health Research (NIHR) Biomedical Research Centre NIHR-BRC-P68711. CC is supported by a personal NIHR Career Development Fellowship (NIHR-2016-090-015). GSC is supported by NIHR Research Professorship. EW is supported by Medical Research Council Project Grant MR/M013278/ 1. AH is supported by NIHR Health Protection Research Unit (grant number HPRU-2012-10, 047) in Healthcare Associated Infections and Antimicrobial Resistance at Imperial College London in partnership with Public Health England. Cerner have not been involved at any stage of this service evaluation, which has been carried out completely independently of them.

\section{AUTHOR CONTRIBUTIONS}

CC and GSC conceived the study. KH, CC, GSC, and AK developed the protocol. AM, BG, MG, AK, CC, and $\mathrm{KH}$ defined the variables of interest from the clinical data and extracted the data. $\mathrm{KH}$ conducted the statistical analysis, which was reviewed and improved by EW and CC. MG and AK, working with the Big Room, refined and developed the data definitions and their clinical relevance and provided ongoing feedback on results and their interpretation. $\mathrm{KH}$ wrote the first draft of the manuscript. All authors reviewed and contributed to the final draft of the manuscript. CC is the guarantor. $\mathrm{KH}$ attests that all listed authors meet authorship criteria and that no others meeting the criteria have been omitted. The views expressed are those of the author(s) and not necessarily those of the National Health Service, National Institute for Health Research, or the Department of Health and Social Care.

\section{SUPPLEMENTARY MATERIAL}

Supplementary material is available at Journal of the American Medical Informatics Association online.

\section{ACKNOWLEDGMENTS}

We thank members of Imperial College NHS Healthcare Trust, specifically the Sepsis Big Room, who participated in the study. We acknowledge the National Institute of Health Research Imperial Biomedical Research Centre who funded this study. The research was conducted using National Institute of Health Research Health Informatics Collaborative data resources. This work was carried out in collaboration with the National Institute for Health Research Health Protection Research Unit in Healthcare Associated Infection and Antimicrobial Resistance at Imperial College London.

\section{CONFLICT OF INTEREST STATEMENT}

AK has received minor hospitality in the form of train travel to a conference and beverages from the Cerner Corporation. Cerner Corporation has not been involved at any stage of this service evaluation, which has been carried out completely independently of them. No other authors declare any competing interests and there are no other relationships or activities that could appear to have influenced the submitted work.

\section{REFERENCES}

1. NHS England. Overview sepsis 2016. https://www.nhs.uk/conditions/sepsis/ Accessed December 28, 2018.

2. Paoli CJ, Reynolds MA, Sinha M, Gitlin M, Crouser E. Epidemiology and costs of sepsis in the united states: an analysis based on timing of diagnosis and severity level. Crit Care Med 2018; 46 (12): 1889-97.

3. Prescott HC. The epidemiology of sepsis. In: Wiersinga WJ, Seymour C, eds. Handbook of Sepsis. Berlin, Germany: Springer; 2018: $15-28$.

4. Reinhart K, Daniels R, Kissoon N, Machado FR, Schachter RD, Finfer S. Recognizing sepsis as a global health priority: a WHO Resolution. N Engl J Med 2017; 377 (5): 414-7.

5. Kumar A, Roberts D, Wood KE, Light B, Parrillo JE, Sharma S. Duration of hypotension before initiation of effective antimicrobial therapy is the critical determinant of survival in human septic shock. Crit Care Med 2006; 34 (6): 1589-96.

6. Ferrer R, Martin-Loeches I, Phillips G, et al. Empiric antibiotic treatment reduces mortality in severe sepsis and septic shock from the first hour: results from a guideline-based performance improvement program. Crit Care Med 2014; 42 (8): 1749-55.

7. Seymour CW, Gesten F, Prescott HC, et al. Time to treatment and mortality during mandated emergency care for sepsis. N Engl J Med 2017; 376 (23): 2235-44.

8. National Health Service. CQUIN Indicator Specification Information on CQUIN 2017/18-2018/19 2019. Gateway Reference 07725. https://www. england.nhs.uk/wp-content/uploads/2017/07/cquin-indicator-specification-information-january-2019.pdf Accessed April 29, 2019.

9. Iacobucci G. NHS hospitals could be fined if they miss new sepsis targets. BMJ 2019; 364: 11124.

10. Singer M, Deutschman CS, Seymour CW, et al. The third international consensus definitions for sepsis and septic shock (sepsis-3). JAMA 2016; 315 (8): 801-10.

11. Royal College of Physicians. National Early Warning Score (NEWS) Standardizing the Assessment of Acute-Illness Severity in the NHS. London, United Kingdom: RCP; 2012.

12. Royal College of Physicians. National Early Warning Score (NEWS) Standardizing the Assessment of Acute-Illness Severity in the NHS . Updated Report of a Working Party. London: RCP; 2017.

13. Gupta T, Puskarich MA, DeVos E, et al. Sequential organ failure assessment component score prediction of in-hospital mortality from sepsis. $J$ Intensive Care Med 2018 Aug 30

14. Goulden R, Hoyle MC, Monis J, et al. qSOFA, SIRS and NEWS for predicting inhospital mortality and ICU admission in emergency admissions treated as sepsis. Emerg Med J 2018; 35 (6): 345-9. 
15. Amland RC, Sutariya BB. Quick sequential [sepsis-related] organ failure assessment (qSOFA) and St. John Sepsis Surveillance Agent to detect patients at risk of sepsis: an observational cohort study. Am J Med Qual 2018; 33 (1): 50-7.

16. Amland RC, Sutariya BB. Saving lives through sepsis surveillance.. https:// www.cerner.com/blog/saving-lives-through-sepsis-surveillance/. Accessed December 28, 2018.

17. Narayanan N, Gross AK, Pintens M, Fee C, MacDougall C. Effect of an electronic medical record alert for severe sepsis among ED patients. Am J Emerg Med 2016; 34 (2): 185-8.

18. Manaktala S, R Claypool SR. Evaluating the impact of a computerized surveillance algorithm and decision support system on sepsis mortality. $J$ Am Med Inform Assoc 2017; 24 (1): 88-95.

19. Ruppel H, Liu V. To catch a killer: electronic sepsis alert tools reaching a fever pitch? BMJ Qual Saf 2019; 28 (9): 693-6.

20. Guirgis FW, Puskarich MA, Smotherman C, et al. Development of a simple sequential organ failure assessment score for risk assessment of emergency department patients with sepsis. J Intensive Care Med 2017 Jan 1.

21. McRee L, Thanavaro JL, Moore K, Goldsmith M, Pasvogel A. The impact of an electronic medical record surveillance program on outcomes for patients with sepsis. Heart Lung 2014; 43 (6): 546-9.

22. Downing NL, Rolnick J, Poole SF, et al. Electronic health record-based clinical decision support alert for severe sepsis: a randomised evaluation. BMJ Qual Saf 2019; 28 (9): 762-8.

23. Silvester KM, Mohammed MA, Harriman P, Girolami A, Downes TW. Timely care for frail older people referred to hospital improves efficiency and reduces mortality without the need for extra resources. Age Ageing 2014; $43(4): 472-7$.

24. Offord N, Harriman P, Downes T. Discharge to assess: transforming the discharge process of frail older patients. Future Hosp J 2017; 4 (1): 30-2.

25. Institute for Healthcare Improvement. Severe Sepsis Bundles 2019. http:// www.ihi.org/resources/Pages/Tools/SevereSepsisBundles.aspx Accessed July 19,2019

26. Amland RC, Hahn-Cover KE. Clinical decision support for early recognition of sepsis. Am J Med Qual 2016; 31 (2): 103-10.
27. Greenhalgh T, Papoutsi C. Spreading and scaling up innovation and improvement. BMJ 2019; 365: 12068.

28. NICE National Institute for Clinical Excellence. Sepsis Quality Standard [QS161]. London: NICE; 2017. https://www.nice.org.uk/guidance/qs161. Accessed December 28, 2018.

29. Austin PC, Stuart EA. Moving towards best practice when using inverse probability of treatment weighting (IPTW) using the propensity score to estimate causal treatment effects in observational studies. Stat Med 2015; 34 (28): 3661-79.

30. Lunceford JK. Stratification and weighting via the propensity score in estimation of causal treatment effects: a comparative study. Stat Med 2017; 36 (14): 2320.

31. NHS Digital. Summary Hospital-level Mortality Indicator (SHMI)-Deaths associated with hospitalization; 2018. https://digital.nhs.uk/data-and-information/publications/clinical-indicators/shmi. Accessed December 28, 2018.

32. Austrian JS, Jamin CT, Doty GR, Blecker S. Impact of an emergency department electronic sepsis surveillance system on patient mortality and length of stay. J Am Med Inform Assoc 2018; 25 (5): 523-9.

33. Shah T, Sterk E, Rech MA. Emergency department sepsis screening tool decreases time to antibiotics in patients with sepsis. Am J Emerg Med 2018; 36 (10): 1745-8.

34. Westra BL, Landman S, Yadav P, Steinbach M. Secondary analysis of an electronic surveillance system combined with multi-focal interventions for early detection of sepsis. Appl Clin Inform 2017; 8 (1): 47-66.

35. Guirgis FW, Jones L, Esma R, et al. Managing sepsis: Electronic recognition, rapid response teams, and standardized care save lives. J Crit Care 2017; 40: 296-302.

36. Craig P, Cooper C, Gunnell D, et al. Using natural experiments to evaluate population health interventions: new Medical Research Council guidance. J Epidemiol Community Health 2012; 66 (12): 1182-6.

37. Inada-Kim M, Nsutebu E. NEWS 2: an opportunity to standardise the management of deterioration and sepsis. BMJ 2018; 360: k1260.

38. Alam N, Hobbelink EL, van Tienhoven AJ, van de Ven PM, Jansma EP, Nanayakkara PW. The impact of the use of the Early Warning Score (EWS) on patient outcomes: a systematic review. Resuscitation 2014; 85 (5): 587-94. 